\title{
Valence- and conduction-band electronic structure of wurtzite CdSe
}

\author{
K. O. Magnusson and S. A. Flodström* \\ $M A X$-Laboratory, University of Lund, Box 118, S-22100 Lund, Sweden
}

(Received 17 October 1986)

\begin{abstract}
The combination of angle-resolved photoelectron and inverse photoelectron spectroscopy has been used to study the electronic band structure of wurtzite CdSe over a 15-eV-wide energy range. Transitions in the uv region involving valence- and conduction-band states along the $\Gamma-M$ line in the hexagonal Brillouin zone are interpreted within a direct-transition model where the final (photoemission) or initial (inverse photoemission) state is approximated by a free-electron state. The energies of four critical points at $M$ and the predicted energy of the optical transition $F_{1}$ are compared with previous theoretical and experimental results.
\end{abstract}

\section{INTRODUCTION}

The optical properties of II-VI compound semiconductors were originally investigated through reflectivity measurements ${ }^{1}$ and these results were then used to calculate the electronic band structure close to the fundamental gap. $^{2,3}$ Presently these properties have again become interesting, now because of interest in the production of optical devices where band-gap modulation through alloying has been exploited. ${ }^{4}$

Photoelectron spectroscopy was first applied to these materials using the angle-integrated technique, ${ }^{5}$ and only recently using angle-resolving spectrometers. ${ }^{6-8}$ Following the experimental work of Stoffel, ${ }^{7}$ Chang, Fröyen, and Cohen calculated the electronic band structure for $\mathrm{CdS},{ }^{9}$ which is the most recent theoretical work of this kind for a wurtzite crystal. II-VI semiconductors have not, to our knowledge, been previously investigated using angleresolved inverse photoelectron spectroscopy.

\section{EXPERIMENT}

The angle-resolved uv photoelectron spectroscopy (ARUPS) experiment was done at the synchrotron radiation laboratory Hasylab, at Desy in Hamburg, the Federal Republic of Germany. The spectrometer, equipped with an electron-energy analyzer mounted on a two-axis goniometer to allow for different polarization geometries, was installed on the Seya-Namioka beam line. ${ }^{10}$ Electron distribution curves (EDC's) were recorded using a multichannel analyzer, with each channel normalized to the photon flux. The total resolution, monochromator plus analyzer, was better than $0.25 \mathrm{eV}$ in all ARUPS spectra.

The angle-resolved inverse photoelectron spectroscopy (INVPES) experiment was performed in a spectrometer at the IBM T.J. Watson Research Center, Yorktown Heights, New York. ${ }^{11}$ Using a highly collimated electron gun with a $\mathrm{BaO}$ filament, a large-acceptance normalincidence spherical grating, and an array of photon counters mounted on the Rowland circle, spectra of the constant-initial-state type, were recorded. Energy and momentum resolutions were typically $0.3 \mathrm{eV}$ and $0.1 \AA^{-1}$, respectively.

Single-crystal rods, $5 \times 5 \times 15 \mathrm{~mm}^{3}$, of low-resistivity
( $\rho \sim 5 \Omega \mathrm{cm})$ CdSe, supplied by Cleveland Crystal Inc., were oriented with the long axis in a [11 $\overline{2} 0]$ direction. (For the indexation of crystal directions in the wurtzite structure, see Ref. 8.) Cleaving under ultrahigh-vacuum conditions, with the standard knife and anvil technique, produced clean but macroscopically stepped (11 20$)$ surfaces.

\section{RESULTS AND DISCUSSION}

Figure 1 presents the normal-emission $k_{\|}=0$, ARUPS spectra from $\mathrm{CdSe}(11 \overline{2} 0)$ recorded for photon energies from 18 to $28 \mathrm{eV}$, with the polarization of the synchrotron radiation perpendicular (left) and parallel (right) to the $c$ axis of the crystal lattice. The polarization geometries were chosen for symmetry reasons since the surface normal and the $c$ axis span a mirror plane of the crystal structure. In these geometries, states along the $\Gamma-M$ line in the

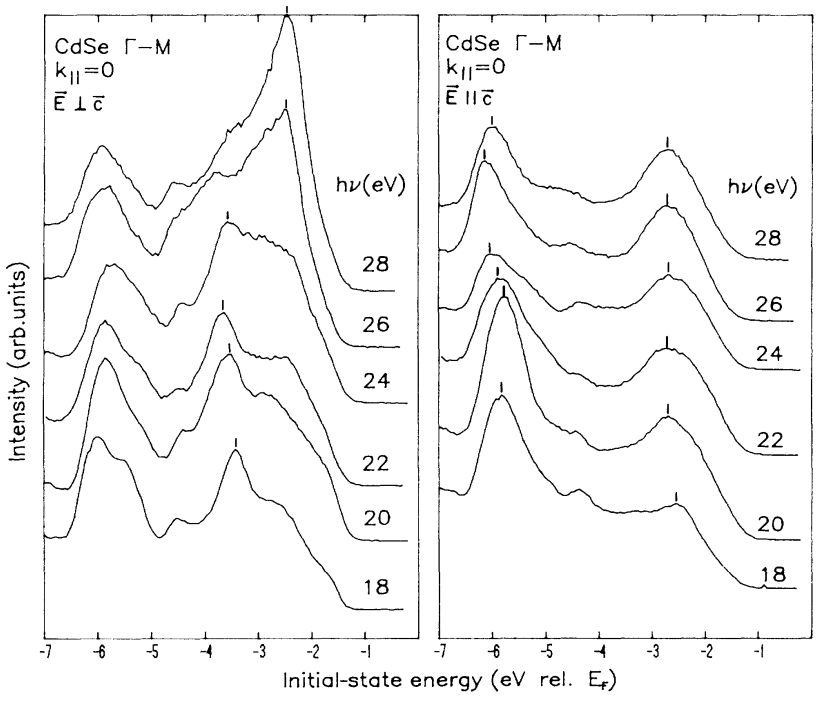

FIG. 1. ARUPS electron distribution curves from the cleaved $\mathrm{CdSe}(11 \overline{2} 0)$ surface recorded in normal emission $k_{\|}=0$ for various photon energies $h v$ and with the polarization perpendicular (left) and parallel (right) to the $c$ axis of the crystal. 
hexagonal Brillouin zone are probed.

In Fig. 1 four structures are indicated. We note that the polarization dependence is striking and that the structures close to the valence-band maximum (VBM) are not well resolved, probably due to the above-mentioned crystal defects in the surface introduced by cleaving.

In addition to the four indicated structures, a weak structure at $\sim 3.3 \mathrm{eV}$ below $E_{F}$ is visible in both polarizations, showing no energy shift with the change in photon energy. The structure around $6 \mathrm{eV}$ below $E_{F}$, observed with the polarization perpendicular to the $c$ axis, is stationary with respect to photon energy, in contrast to the structure in this initial-energy region observed in the second polarization geometry. In off-normal measurements the stationary structure has also been found to be stationary in initial energy with respect to angle of emission. We assign this structure to emission from nondirect transitions. A similar structure is also observed for $\mathrm{CdS}$. $^{7,8}$

The results of the INVPES experiment are shown in Fig. 2. The spectra are for normally incident electrons $k_{\|}=0$ with initial-state energies from 10.5 to $21.25 \mathrm{eV}$, probing states along the $\Gamma-M$ line in the Brillouin zone. Several structures are present and it is notable that some of them are quite broad and that the structures show only small shifts in final-state energy with the change in electron initial-state energy. The observed structures are broadened by the finite lifetimes in the excited states, and for some structures the impossibility of distinguishing the

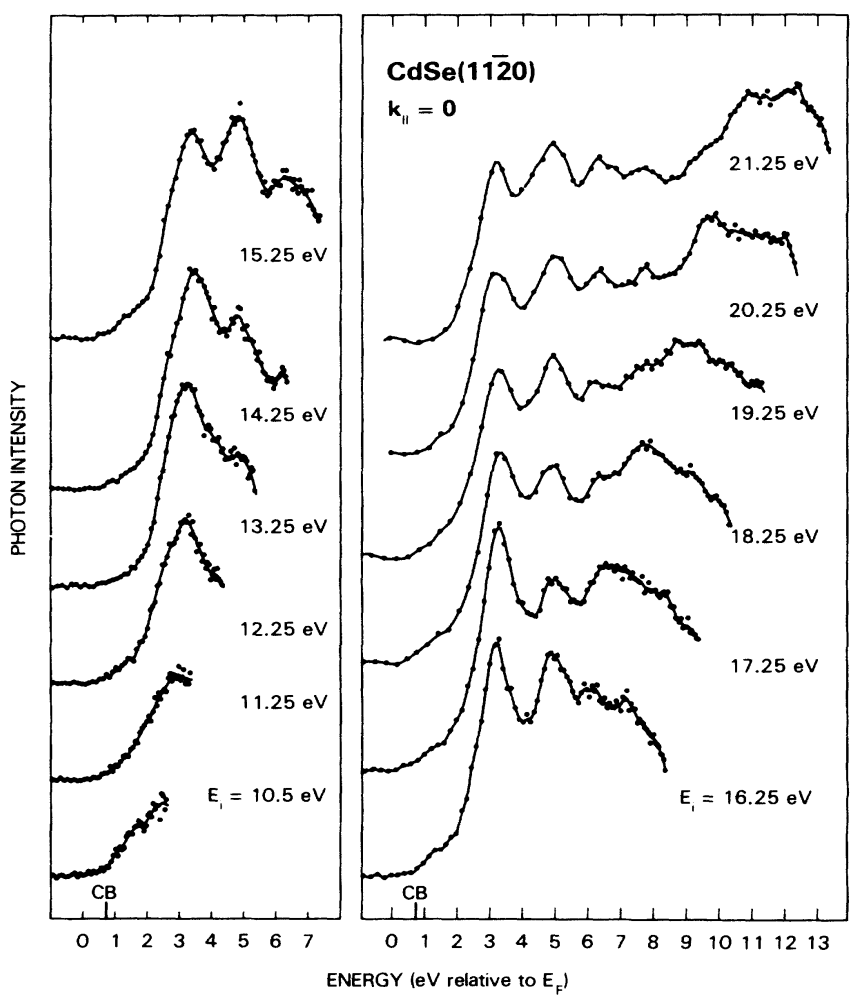

FIG. 2. INVPES constant initial-state spectra recorded for various initial-state energies $E_{i}$ of the electrons impinging at normal incidence $k_{\|}=0$ on the cleaved (1120) surface of CdSe. polarization of the photons results in unresolved, closely spaced bands of different symmetry with respect to the mirror plane.

The results presented in Figs. 1 and 2 are interpreted within a model of momentum-conserving direct transitions between the valence (ARUPS) or conduction (INVPES) bands and higher-lying states. Electronic states $8 \mathrm{eV}$ above the VBM have not been available from calculations for $\mathrm{CdSe}$ and we have chosen to approximate these states with a free-electron parabolic state

$$
E(k)=(\hbar / 2 m)^{2}(\mathbf{k}+\mathbf{G})^{2}+E_{0} .
$$

The band bottom $E_{0}$ is determined from a best-fit procedure for the ARUPS results to the calculated bands. We have used the calculation by Chang et al. ${ }^{9}$ which, despite the fact that it was performed for CdS, gives a better agreement with experiment than does the calculation for CdSe by Bergstresser and Cohen. ${ }^{2}$ The reason for this is probably the difference in experimental information at the time of calculation and the similarity between the band structure of CdS and CdSe, as stated in Ref. 2. Fitting the dispersion of the lowest-lying valence band, we find the band bottom, $E_{0}=-4.0 \mathrm{eV}$.

Of great importance in combining ARUPS and INVPES results is finding a common reference energy. The INVPES spectrometer was calibrated to assign a final-state energy of $0 \mathrm{eV}$ to transitions down to the Fermi level. Through measurement of the contact potential with a Kelvin probe and using a band gap of $1.98 \mathrm{eV}$ and a photothreshold of $6.6 \mathrm{eV}$, we find the energy position of the VBM. In the ARUPS experiment the Cd $4 d_{5 / 2}$ level was used as a reference with a binding energy of $9.79 \mathrm{eV}$ with respect to the VBM. ${ }^{12,13}$

Figure 3 presents the results of the experiments together with the calculated bands from Ref. 9. The ARUPS results are indicated with circles for structures observed with the polarization of the light perpendicular to the $c$ axis of the crystal and with triangles for structures observed with the polarization parallel to the $c$ axis. The structures observed in the INVPES experiment are marked with squares. All open symbols denote weaker structures. The solid lines are the calculated bands, linearly contracted due to the larger unit cell of CdSe and adjusted to give the correct band gap for CdSe by a rigid shift of the conduction bands. The experimental points have been derived using a single (normal-direction) free-electron parabolic state for the final state in the ARUPS experiment as well as for the initial state in the INVPES experiment.

In the conduction band the second, third, and probably the overlapping contributions from the fourth and fifth band, numerated from the fundamental band gap and up, are observed. Indicated as a weaker structure we find also a contribution from a state lying higher in energy than the maximum energy for which the calculation was performed. A weak dispersive behavior is found mainly for the lowest observed conduction band.

Two strong structures in the observed valence band are clearly dispersing in the part of the Brillouin zone studied. From symmetry arguments we expect to see contributions only from the first and the third of the valence bands, counting from the fundamental band gap down at $M$, 


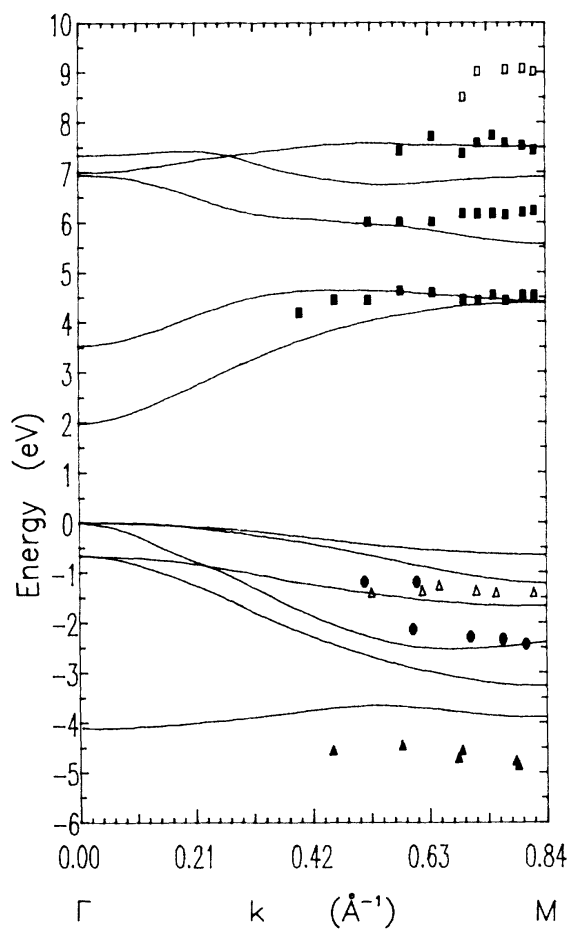

FIG. 3. The experimental electronic band structure of $\mathrm{CdSe}$ along the $\Gamma-M$ line. The results of the ARUPS experiment are presented as circles ( $E$ perpendicular to $\mathbf{c}$ ) and triangles (E parallel with $\mathbf{c}$ ), while the results of the INVPES experiment are presented as squares. All open symbols denote weaker structures. The interpretation is based on a free-electron upper state with $E_{0}=-4.0 \mathrm{eV}$. The solid lines are the calculated bands of CdS from Chang et al. (Ref. 9), adjusted to give the correct band gap and Brillouin zone size for CdSe. Energies are relative to the valence-band maximum.

when the polarization is perpendicular to the $c$ axis. The remaining bands in the energy region studied should be observed with the polarization parallel with the $c$ axis. From this we conclude that the experimental band about $2.5 \mathrm{eV}$ below the VBM at $M$ is the third valence band from the top, and the experimental band about $5 \mathrm{eV}$ below the VBM at $M$ is the sixth valence band from the top. This last band has a local maximum of $E_{i}=-4.5 \pm 0.1 \mathrm{eV}$ at $k \approx 0.6 \AA^{-1}$, in agreement with the shape of the corresponding calculated band from Ref. 9. The two remaining observed structures in this initial-energy region have not been accurately identified.

With the above-presented information, four critical points at $M$ are determined. Table I presents a compilation of the calculated energies from Refs. 2 and 9 and the energies observed in this study. Furthermore, the energy of the $F_{1}$ structure observed in reflectivity measurements by Cardona and Harbeke ${ }^{1}$ is compared with the theoretically predicted value from Bergstresser and Cohen, ${ }^{2}$ and the value derived from the work presented in this paper. The $F_{1}$ structure is only observed when the polarization of the light is perpendicular to the $c$ axis of the crystal, in agreement with the polarization geometry found to excite
TABLE I. Energy positions relative to the VBM of critical points at $M$ in the Brillouin zone for CdSe. Values from Bergstresser and Cohen (Ref. 2) (from the band structure plot) and Chang et al. (Ref. 9) are presented together with the results of the present work. The predicted energy of the reflectivity structure $F_{1}$ from Bergstresser and Cohen is also presented together with the observed reflectivity peak energy from Cardona and Horbeke (Ref. 1) and the experimentally predicted energy found in this work.

\begin{tabular}{|c|c|c|c|}
\hline \multirow[b]{2}{*}{$\begin{array}{c}\text { Critical } \\
\text { point }\end{array}$} & \multicolumn{3}{|c|}{ Energy relative to VBM $(\mathrm{eV})$} \\
\hline & Bergstresser & Chang & $\begin{array}{c}\text { Present } \\
\text { work }\end{array}$ \\
\hline$M_{3}$ & 6.7 & 5.42 & $6.25 \pm 0.15$ \\
\hline$M_{1}$ & 5.1 & 4.27 & $4.55 \pm 0.15$ \\
\hline$M_{2}$ & -1.1 & -1.7 & $-2.45 \pm 0.10$ \\
\hline$M_{1}$ & -2.5 & -3.9 & $-4.90 \pm 0.10$ \\
\hline \multirow[b]{2}{*}{$\begin{array}{l}\text { Reflectivity } \\
\text { structure }\end{array}$} & \multicolumn{3}{|c|}{ Energy $(\mathrm{eV})$} \\
\hline & Bergstresser & Cardona & $\begin{array}{l}\text { Present } \\
\text { work }\end{array}$ \\
\hline$F_{1}: M_{2} \rightarrow M_{1}$ & 6.2 & 6.8 & $7.0 \pm 0.15$ \\
\hline
\end{tabular}

the photoelectron transition, which in this work was used to derive the energy value for $F_{1}$.

\section{SUMMARY}

We have demonstrated the power of combining ARUPS results with INVPES results in the study of bulk electronic structure. By tuning the photon energy in ARUPS and the electron kinetic energy in INVPES, information from selected points in the Brillouin zone is obtainable. From the determination of critical points in the filled valence band, as well as in the unfilled conduction band, the energy splitting between states involved in optical transitions is found. In contrast to, e.g., reflectivity measurements, here, in principle, we find all transitions at, e.g., the $M$ point, not only the transition which is strong enough to dominate over all other transitions, within the energy range in question, throughout the Brillouin zone. This is in addition to the basic difference in the possibilities of photoelectron and inverse photoelectron spectrsocopy to determine absolute electronic energies.

In the present study, four critical points in the band structure of $\mathrm{CdSe}$ have been determined, thus giving the absolute energy position relative to the VBM for these bands. Obtaining a mutual energy reference for the two experiments with the scheme used is proved satisfactory through the comparison of the $M_{2}-M_{1}$ splitting experimentally observed by Cardona and Harbeke ${ }^{1}$ with the result derived from our work.

The calculated band structure for $\mathrm{CdSe}^{2}$ is far from satisfactory in predicting the critical point energies, far worse than what is apparent from a comparison with the reflectivity experiment. ${ }^{1}$ We would welcome a new calculation, perhaps of the type presented for CdS in Ref. 9. 


\section{ACKNOWLEDGMENTS}

The authors are indebted to Dr. M. Lähdeniemi and the Hasylab staff for providing the opportunity to perform the experiment and to P. Mårtensson and S. Wiklund for their assistance. The support from Dr. F. J. Himpsel and the IBM T.J. Watson Research Center and the assistance of Dr. U. O. Karlsson and Dr. D. Straub is gratefully acknowledged. This work was supported by the Swedish Natural Science Research Council and by a grant from the Knut and Alice Wallenberg Foundation.

*Present address: Physics III, Royal Institute of Technology, S10044 Stockholm, Sweden.

${ }^{1}$ M. Cardona and G. Harbeke, Phys. Rev. 137, A1467 (1965).

${ }^{2}$ T. K. Bergstresser and M. L. Cohen, Phys. Rev. 164, 1069 (1967).

${ }^{3}$ R. N. Euwema, T. C. Collins, D. G. Shankland, and J. S. DeWitt, Phys. Rev. 162, 710 (1967).

${ }^{4}$ See, e.g., A.-B. Chen and A. Sher, J. Vac. Sci. Technol. 21, 138 (1982).

${ }^{5}$ N. B. Kindig and W. E. Spicer, Phys. Rev. 138, A561 (1965).

${ }^{6}$ A. Ebina, T. Unno, Y. Suda, H. Koinuma, and T. Takahashi, J. Vac. Sci. Technol. 19, 301 (1981).

${ }^{7}$ N. G. Stoffel, Phys. Rev. B 28, 3306 (1983).
${ }^{8}$ K. O. Magnusson, S. A. Flodström, P. Mårtensson, J. M. Nicholls, U. O. Karlsson, R. Engelhardt, and E. E. Koch, Solid State Commun. 55, 643 (1985).

${ }^{9}$ K. J. Chang, S. Fröyen, and M. L. Cohen, Phys. Rev. B 28, 4736 (1983).

${ }^{10}$ C. A. Feldman, R. Engelhardt, T. Permien, E. E. Koch, and V. Saile, Nucl. Instrum. Methods 208, 785 (1983).

${ }^{11}$ Th. Fauster, D. Straub, J. J. Donelon, D. Grimm, A. Marx, and F. J. Himpsel, Rev. Sci. Instrum. 56, 1212 (1985).

${ }^{12}$ L. Ley, R. A. Pollak, F. R. McFeely, S. P. Kowalczyk, and D. A. Shirley, Phys. Rev. B 9, 600 (1974).

${ }^{13}$ A. Ebina, K. Asano, and T. Takahashi, Phys. Rev. B 22, 1980 (1980). 